\title{
Twelve-Month Efficacy of an Obesity Prevention Program Targeting Hispanic Families With Preschoolers From Low-Income Backgrounds
}

Sheryl O. Hughes, $\mathrm{PhD}^{1}$; Thomas G. Power, $\mathrm{PhD}^{2}$; Ashley D. Beck, $\mathrm{PhD}^{3}$; Drew Betz, $\mathrm{MS}^{4}$; L. Suzanne Goodell, $\mathrm{PhD}, \mathrm{RD}^{5}$; Veronica Hopwood, $\mathrm{PhD}^{6}$; J. Andrea Jaramillo, $\mathrm{MA}^{1}$; Jane Lanigan, $\mathrm{PhD}^{7}$; AnaMaria Diaz Martinez, $\mathrm{Med}^{8}$; Nilda Micheli, $\mathrm{BS}^{1}$; Yadira Olivera Guerrero, $\mathrm{PhD}^{9}$; Irene Overath, MACEd ${ }^{10}$; Louise Parker, $\mathrm{PhD}^{11}$; Guadalupe Ramos, $\mathrm{PhD}^{12}$; Yuri P. Thompson, $\mathrm{MS}^{13}$; Maria A. Papaioannou, $\mathrm{BS}^{1}$; Susan L. Johnson, $\mathrm{PhD}^{14}$

\begin{abstract}
Objective: Assess effects of an obesity prevention program promoting eating self-regulation and healthy preferences in Hispanic preschool children.

Design: Randomized controlled trial with pretest, posttest, 6- and 12-month assessments. Fourteen waves, each lasting 7 weeks.

Setting: Families recruited from Head Start across 2 sites.

Participants: Two hundred fifty-five families randomized into prevention $(\mathrm{n}=136)$ or control $(\mathrm{n}=119)$.

Intervention: Prevention received curriculum; control received no curriculum.

Main Outcome Measure(s): Feeding knowledge/practices/styles (parent); body mass index percentile, eating self-regulation, trying new foods, and fruit/vegetable consumption (child).

Analysis: Multilevel analyses for nested data (time points within families; families within waves) and multinomial regression.

Results: Program increased mothers' repeated presentation of new foods $(P<0.05)$, measured portion sizes $(P<0.05)$, child involvement in food preparation $(P<0.001)$, feeding responsiveness $(P<0.001)$, knowledge of best feeding practices $(P<0.001)$, and feeding efficacy $(P<0.05)$; reduced feeding misconceptions $(P<0.01)$ and uninvolved feeding $(P<0.01)$. Effects on child eating behavior were minimal. At 12 months, children in the prevention group were less likely to have overweight $(P<0.05)$ or obesity $(P<0.05)$.
\end{abstract}

\footnotetext{
${ }^{1}$ USDA/ARS Children's Nutrition Research Center, Department of Pediatrics, Baylor College of Medicine, Houston, TX

${ }^{2}$ Department of Human Development, Washington State University, Pullman, WA

${ }^{3}$ Spokane Regional Health District, Spokane, WA

${ }^{4}$ Whatcom County Extension, Washington State University, Bellingham, WA

${ }^{5}$ Department of Food, Bioprocessing, and Nutrition Sciences, North Carolina State University, Raleigh, NC

${ }^{6}$ Pullman Regional Hospital, Pullman, WA

${ }^{7}$ Department of Human Development, Washington State University, Vancouver, WA

${ }^{8}$ Franklin County Extension, Washington State University, Pasco, WA

${ }^{9}$ Yakima County Extension, Washington State University, Union Gap, WA

${ }^{10}$ Kitsap County Extension, Washington State University, Bremerton, WA

${ }^{11}$ Department of Human Development, Washington State University Extension, Seattle, WA

${ }^{12}$ Department of Preventive Medicine, University of Southern California, Los Angeles, CA

${ }^{13}$ College of Education, Department of Psychological, Health, and Learning Sciences, University of Houston, Houston, TX

${ }^{14}$ Department of Pediatrics, Section of Nutrition, University of Colorado School of Medicine, Aurora, CO

Conflict of Interest Disclosure: The last author of this article (S. L. Johnson) serves on the JNEB staff as Associate Editor. Review of this article was handled, exclusively, by the Editor-in-Chief to minimize conflict of interest. The rest of the authors have not stated any conflicts of interest.

Address for correspondence: Sheryl O. Hughes, PhD, USDA/ARS Children's Nutrition Research Center, Baylor College of Medicine, 1100 Bates Ave, Houston, TX; E-mail: shughes@bcm.edu

(C) 2021 Society for Nutrition Education and Behavior. Published by Elsevier Inc. All rights reserved.

https://doi.org/10.1016/j.jneb.2021.04.460
} 
Conclusions and Implications: Program effects emphasize the importance of feeding approaches in reducing childhood obesity.

Key Words: childhood obesity, prevention program, family-based, child eating self-regulation, child fruit and vegetable consumption (J Nutr Educ Behav. 2021;53:677-690.)

Accepted April 19, 2021. Published online June 18, 2021.

\section{INTRODUCTION}

Overweight and obesity in children continue to pose a very serious public health threat in the US. ${ }^{1}$ About 35\% of US children and youth are currently overweight or obese. ${ }^{1}$ Among younger children (aged 2-5 years), just under a quarter of the children are overweight or obese, ${ }^{2}$ with Hispanic children having the highest rates at $30 \%{ }^{3}$ Given the importance of the first 5 years of life in the development of child eating behaviors, ${ }^{4}$ targeting Hispanic preschoolers in obesity prevention programs may prove fruitful.

A wide range of prevention programs have been developed to prevent overweight and obesity in children and youth. ${ }^{5,6}$ Most programs have met with minimal success, especially when examining long-term outcomes on child weight status. ${ }^{5-7}$ Prevention programs targeting preschoolers have shown similar results with limited impact on the weight status of the children. ${ }^{8}$ Moreover, only a few successful obesity prevention programs for children and youth have been specifically designed for ethnically diverse populations with low incomes. ${ }^{9}$

Families play an influential role in the development of behaviors that both foster and reduce the likelihood of childhood obesity. ${ }^{10}$ Participation of family members (especially parents) has been targeted as an essential component in obesity prevention programs, particularly among younger children who are in the process of developing eating preferences and may be responsive to adopting new habits and patterns. ${ }^{11} \mathrm{~A}$ recent systematic review of family-based obesity prevention programs identified 6 randomized controlled trials targeting Hispanic families. ${ }^{12}$ Of these studies, only 2 were designed for younger children aged 2-5 years of age, $^{13,14}$ and only 1 was an obesity prevention program (all children in Foster et $\mathrm{al}^{14}$ were already obese). Although several obesity-related risk factors were targeted in these 2 family-based studies designed for Hispanic preschoolers, only the obesity treatment program ${ }^{14}$ showed changes in child body mass index (BMI). Given that family-based obesity prevention programs are limited, and only a small number of programs target ethnically diverse families, ${ }^{12}$ the Strategies for Effective Eating Development (SEEDS) obesity prevention program was developed for parents and their preschool-aged children from families with low incomes. ${ }^{15}$

The SEEDS prevention program teaches parents and children to pay attention to children's internal cues of hunger and fullness and to increase children's willingness to try new foods. Also included are strategies for promoting appropriate child portion sizes, tactics for encouraging family routines and structure, and information for dealing with outside influences on family eating. The program includes 7 lessons delivered to families sequentially over 7 weeks. As part of the 7 curriculum lessons, videos and experiential learning activities reinforce the information presented. The development of the curriculum, videos, and activities are described in 2 previous publications. ${ }^{15,16}$ A detailed description of the lessons can be found in Table 1 of the SEEDS research methods publication. ${ }^{15}$

The program was developed for parents of preschool children from low-income backgrounds. Focus groups of African American and Hispanic parents provided information on attitudes, beliefs, and practices regarding the feeding of young children. ${ }^{19}$ Although culturally specific content was not built into the lessons, the videos incorporated diverse family groups, identifiable settings, and a wide variety of foods that are culturally relevant. Furthermore, the discussion-based format of the lessons allowed facilitators and parents to discuss culturally relevant examples and issues while adhering to the core content of the program. The focus was on Hispanic parents and their children for this first efficacy trial because of the high risk for childhood obesity in this population and the need to initially test the program with a relatively homogenous sample.

This article reports on the 12month efficacy of the SEEDS obesity prevention program using a randomized controlled design. Three overall outcome areas were targeted: parental feeding, child eating behaviors, and child weight status. Regarding parental feeding, it was predicted that prevention parents compared with controls would be more likely to (1) encourage child exploration of new foods, (2) use more child-centered feeding practices (eg, responsivity to fullness cues), and less parentcentered feeding practices (eg, pressuring the child to eat), (3) provide more structure during mealtimes, (4) show more authoritative feeding styles (ie, encourage child eating using nondirective and supporting behaviors); and (5) demonstrate higher levels of best practices feeding knowledge. Regarding child eating behaviors, it was expected that prevention children compared with controls would be more likely to show: (1) increased self-regulation of energy intake, (2) more willingness to try new foods, and (3) greater consumption of fruit and vegetables (including a wider variety of fruit and vegetables). All effects were expected to continue through the 6- and 12month follow-ups. Regarding child weight status, it was expected that decreases in child BMI percentiles would be seen by the 6- and 12month follow-ups for the prevention group compared with the control group (Figure 1). 
Table 1. Weight Status and Demographic Characteristics of Mothers and Children at Pretest (\%)

\section{Characteristics}

Mother and child characteristics

Child sex, female

Child age, mo, mean (SD)*

Mother age, y, mean (SD)

Mothers' place of birth*

Central America

Mexico

US

Other (South America, Puerto Rico)

Not reported

Highest level of maternal education*

Sixth grade or less

Eighth grade or less

Attended some high school

High school graduate or GED

Completed technical school

Attended some college

College graduate

Marital status

Married

Never married

Widowed, separated, divorced, other

Employment status, currently employed

Child BMI categories $^{17}$

Normal (<85th percentile)

Overweight (85th to 95th percentile)

Obese ( $\geq 95$ th percentile)

Maternal BMI categories $\left(\mathrm{kg} / \mathrm{m}^{2}\right)^{18}$

Underweight $(<5)$

Normal $(<25)$

Overweight ( $\geq 25$ to $<30$ )

Obese $(\geq 30)$
Urban City (112 Mother/

Child Dyads)

55.4

$53.5(6.9)$

$33.8(7.3)$

18.7

62.5

12.5

3.6

2.7

12.5

10.7

16.1

18.8

14.3

12.5

15.2

60.7

15.2

24.1

33.9

69.6

14.3

16.1

1.8

11.7

39.6

46.8
Agricultural Community (143 Mother/Child Dyads)

44.8

$57.0(6.7)$

$32.9(6.8)$

1.4

88.8

7.0

0

2.8

29.2

17.5

6.6

30.7

6.6

3.6

5.8

65.0

16.8

18.2

42.6

56.8

23.0

20.1

1.4

17.2

30.7

50.7

BMI indicates body mass index; GED, general education development.

${ }^{*} P<0.001$.

Note: Pearson chi-square tests were used for child sex, mother's place of birth, highest level of education, marital status, child $\mathrm{BMI}$, and maternal BMI t tests were used for chld age and mother age.

\section{METHODS}

\section{Study Design}

A randomized controlled trial was implemented across 2 sites (Houston, an urban city in Texas; and Pasco, an agricultural community in Washington) to examine the efficacy of the SEEDS childhood obesity prevention program. The prevention arm received parent and child program curricula separately over 7 weeks for a total of 7 lessons. One lesson was held each week, with parent and child lessons held simultaneously. Each lesson lasted about an hour. A combined family lesson (parent and child together) was held before the parent and child lessons. All lessons were held after the school day in Spanish at Head Start centers in Houston, TX, and similar early education centers in Pasco, WA (more details regarding the centers can be found under participant recruitment). The control arm received no curriculum. Approximately 8-10 families participated in each of the prevention and control conditions at each wave. Fourteen waves were conducted (6 in Houston and 8 in Pasco) between 2014 and 2016. Parents were asked if they could read and write in English or Spanish during eligibility screening so that assistance would be made available at the data collection sessions for those who needed help completing the questionnaires. Participants in the prevention and control arms completed the same assessments before the prevention program (pretest), after the program (posttest), and at 6- and 12-month follow-ups. Compensation was $\$ 40$ at pretest, $\$ 50$ at posttest, $\$ 60$ at 6 months, and $\$ 85$ at 12 months.

\section{Facilitators}

Bilingual staff members led the faceto-face groups (ie, parent, child, and family lessons). Parent facilitators were required to have a graduate 


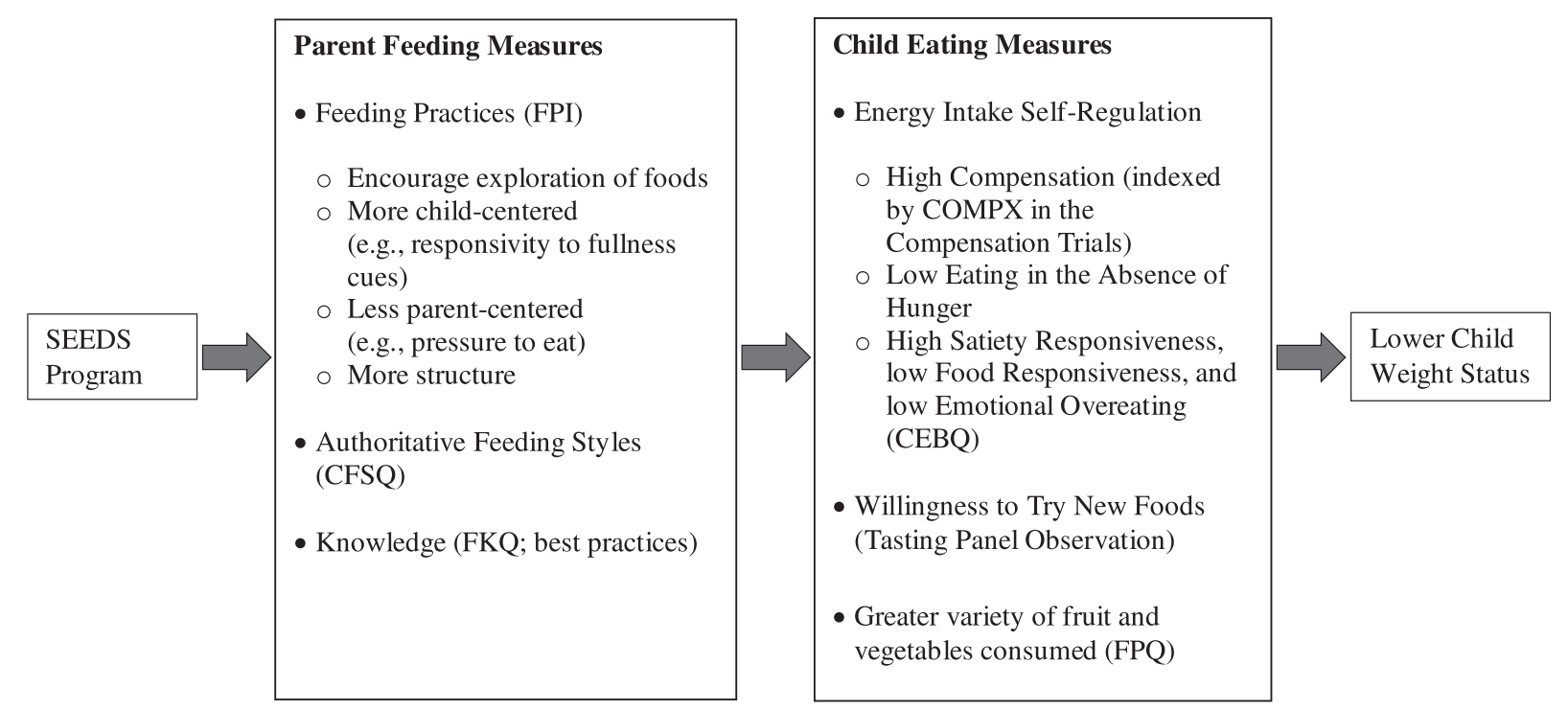

Figure 1. Conceptual model: hypotheses and measures. CEBQ indicates Children's Eating Behavior Questionnaire; CFSQ, Caregiver's Feeding Styles Questionnaire; FKQ, Feeding Knowledge Questionnaire; FPI, Food Parenting Inventory; FPQ, Food Preferences Questionnaire; SEEDS, Strategies for Effective Eating Development.

degree in psychology or education to optimize behavior change that was integrated into the program content. Child facilitators were required to have training or experience in early childhood education. Family lessons were co-facilitated by the parent and child facilitators. Facilitator training included a 2-day intensive training session guided by study investigators, which included coverage of the program content, delivery of the lessons in practice sessions, and training to maximize participant engagement and involvement on the basis of educational principles. These principles were reinforced during 2 follow-up training sessions held for the facilitators throughout the randomized controlled trial. These follow-up training sessions included maintaining intervention fidelity and discussions regarding challenging questions or comments from parents that the facilitators experienced and ways to address them.

\section{Participants}

Families (parent/child dyads) were recruited from Head Start centers in Houston, TX, and child care centers serving families with low incomes in Pasco, WA. The Pasco centers were similar to Head Start in that they provided free services and support for families with low incomes. The goal of these centers was to ensure that all children enter kindergarten ready to succeed. $^{20}$ Children who were 3 -5 years of age with their parents identifying as Hispanic were considered eligible. Exclusion criteria were as follows: parent aged under 18 years and parent and/or child had major food allergies, diabetes, or were on special diets, including those who were vegetarian. A total of 255 Hispanic parent/child dyads were enrolled in the study at pretest. On completion of pretest assessments, the project coordinator at each site used a computer-based randomization tool to assign participants to 1 of 2 arms using simple randomization (136 prevention; 119 control). The retention rates (see Figure 2) were $84 \%$ at posttest $(n=214), 66 \%$ at 6 months $(n=169)$, and $61 \%$ at 12 months $(n=156)$. Recruitment methods, as well as sample size calculations, are described in more detail in previous publications. ${ }^{15,16}$ The study was reviewed and approved by the Institutional Review Boards at Baylor College of Medicine and Washington State University. Parents provided informed consent for themselves and their children before taking part in the study, and assent was obtained from the children. Most parents recruited were mothers (99\%) with a small number of grandmothers. All parents are referred to as mothers below.

\section{Program Fidelity}

Observers trained in the curriculum content conducted fidelity observations on $39 \%$ of the lessons. On the basis of observer availability, the observations were distributed across parent (36\%), child (34\%), and family $(30 \%)$ sessions. Facilitators were not notified in advance that they would be observed. Observers were different from the facilitators delivering the curriculum. Fidelity was defined as the number of yes responses reported by the observer (signifying that the facilitator adhered to the lesson component as described in the curriculum) divided by the total number of possible responses (ie, yes, some, and no). Across the parent, child, and family lessons, fidelity ranged from $67 \%$ to $100 \%$, with a mean of $95 \%$. Despite this wide range, $85 \%$ of the lessons had fidelity values greater than $85 \%$.

\section{Measures}

As described in previous publications, ${ }^{15,16}$ data collections for both the prevention and control arms were conducted in small group 


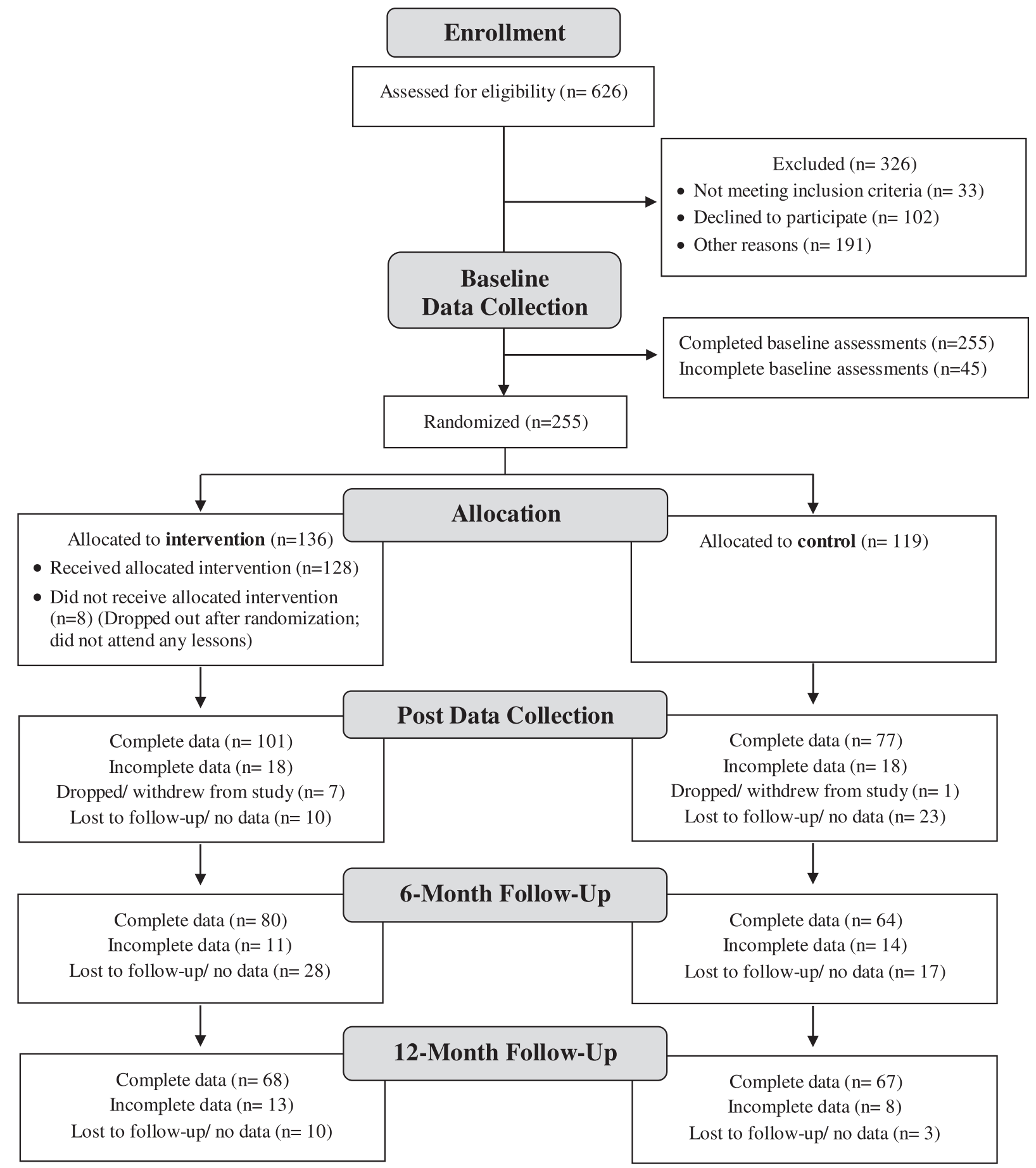

Figure 2. Strategies for Effective Eating Development (SEEDS) Consort Flow Diagram.

settings led by trained staff members of Hispanic descent who were proficient in reading, speaking, and writing in English and Spanish. Data collectors were blinded to participant group allocation. For mothers with low literacy skills, data collectors read the questions to the mothers and recorded their responses. Child assessments were completed at the same time as the mothers in a separate room. Data collection measures are briefly described below; more detailed information on the measures can be found elsewhere. ${ }^{15,16}$ Most measures were validated for use with preschoolers and Hispanic samples. 
Parent Feeding Measures

(Practices, Styles, and

Knowledge)

Food Parenting Inventory. The Food Parenting Inventory (FPI) is a parentreport measure of feeding practices with 16 subscales assessing 3 higher level feeding constructs: (1) encourage trying new foods (offer new foods, encourage exploration of new foods, urge the child to eat new foods, and repeat presentation of new foods); (2) mealtime structure (family meals, regular timing of meals and snacks, inconsistent mealtimes, indifferent feeding, child involvement in food preparation, the parent decides portion sizes, and serving measured portions); and (3) external control (pressure to eat, restriction, food as reward, responsiveness to child's fullness cues, and monitoring). ${ }^{21}$ Preliminary analyses of baseline data for 254 of the Hispanic mothers in the current sample showed excellent reliability and validity for this instrument. See Power et $\mathrm{al}^{21}$ for more details.

Caregiver's Feeding Styles Questionnaire. The Caregiver's Feeding Styles Questionnaire (CFSQ) is a parent-report questionnaire developed to measure feeding styles in parents with low incomes. $^{22}$ The questionnaire was developed for use with parents of children aged 3-11 years. Parents indicate how often they use 7 child-centered (eg, reasoning, complimenting) and 12 parent-centered (eg, demands, threats) feeding directives. Two feeding style dimension scores of demandingness and responsiveness are derived from the child-centered and parent-centered items. Parents are classified into 4 feeding style categories of authoritarian, authoritative, indulgent, and uninvolved. Evidence of test-retest reliability, internal consistency, convergent and predictive validity has been demonstrated in Hispanic samples and samples with low incomes. ${ }^{22-24}$

Feeding Knowledge Questionnaire. The Feeding Knowledge Questionnaire (FKQ) was developed for the current study to assess the degree to which mothers learned the program content. Forty items measured the main messages of the program. Sixteen items referred to best practices feeding knowledge, 11 items referred to common misconceptions about feeding, and 11 questions measured mothers' efficacy in feeding their child. Other program content was measured: (1) exposure to new foods and (2) child and parent roles during feeding. See Hughes et $\mathrm{al}^{16}$ for more detailed information on this questionnaire.

\section{Children's Eating Behavior Measures (Self-regulation, Trying New Foods, and Fruit and Vegetable Consumption)}

Compensation protocol. Observed trials of children's response to changes in energy density in a meal (compensation trials) were used to assess child eating self-regulation. ${ }^{25}$ On 2 separate days, children participated in a 2-part meal consisting of 1 of 2 versions of a drink preload and a standard meal. On each day, the drink preload was either high (152 kcal) or low (3 kcal) in energy. The standard meal, containing about 595 calories or $40 \%$ of the daily food requirements for children aged 3-5 years, was served 30 minutes later. Consumption was measured by weighing the foods before and after the standard meal on the basis of manufacturers' information. ${ }^{25,26}$ An eating self-regulation score (COMPX) was calculated by determining how each child adjusted his/her intake at the meal on the basis of the 2 preloads. This score has been used successfully to evaluate an intervention to improve children's self-regulation of energy intake. ${ }^{27}$ This measure has been used with preschool-aged children $^{28}$; to the authors' knowledge, it has not been used previously with Hispanic samples.

Eating in the absence of hunger. Child eating self-regulation was also measured by the eating in the absence of hunger (EAH) task developed by Fisher and Birch. ${ }^{29}$ On the day the child received the high-energy preload drink as part of the compensation task, and approximately 10-15 minutes after finishing the standard meal, the child was presented with sweet and savory snacks along with age-appropriate toys. Children were left with the food and toys for $10 \mathrm{mi}$ nutes and told they could eat what they wanted, sit quietly, and/or play with the toys. This task was administered in the centers to several children at once, although children completed the task individually through the use of portable screens, creating a separate assessment area for each child. The remaining snacks were measured, and the total kcal consumed was calculated. Scores reflected the total number of kcal eaten in the absence of hunger. This assessment has been used predominately with White children and has been validated with preschoolers; a few studies have used the EAH task with Hispanic children (see Lansigan et $\mathrm{al}^{30}$ for a review).

Children's Eating Behavior Questionnaire. The Children's Eating Behavior Questionnaire (CEBQ) is a parentreport measure assessing 8 dimensions of child eating behaviors. ${ }^{31}$ Validity and reliability have been demonstrated. ${ }^{31}$ For this study, only the subscales of food responsiveness, emotional overeating, and satiety responsiveness were targeted because they are considered parent-report measures of child eating behaviors related to eating self-regulation. The CEBQ has shown adequate validity and reliability ${ }^{31}$ and has been used successfully with Hispanic parents of preschoolers with lowincome levels. $^{32}$

Child tasting panel observation. A standardized food tasting protocol, developed by Sullivan and Birch ${ }^{33}$ and modified by Moding, Bellows, Grimm, and Johnson, ${ }^{34}$ was administered to measure taste preferences (Yummy, Just OK, or Yucky) and children's willingness to try new foods $(1=$ tried; $0=$ refused $)$. Each child was asked to try bite-sized samples of 9 familiar and novel foods from a variety of food groups, including broccoli, beets, couscous, garbanzo beans, gouda cheese, grapefruit, kale, okra, and papaya. Each food was served in a small 2-oz cup 
and included 3 small pieces of the food. This assessment tool was developed for a large, 3-year longitudinal study of preschoolers in Colorado (41\% Hispanic). ${ }^{34}$

Food Preferences Questionnaire. The variety of fruit and vegetables consumed by the children was measured by a Food Preference Questionnaire (FPQ) adapted from Skinner and colleagues. ${ }^{35}$ Parents reported on their child's food consumption from 112 food and drink items using 3 categories: never tried it, tried - liked it, and tried - did not like it. Similar measures have been used successfully by the Food and Drug Administration in the Total Diet Study. ${ }^{35}$ Only data on the fruit and vegetables were analyzed given the program emphasis on trying fruit and vegetables and the fact that the vast majority of foods in the questionnaire were fruit and vegetables. Two measures were examined: the total number of vegetables tried out of 50 , and the total number of fruits tried out of 27 . This questionnaire has been used predominately with White samples. ${ }^{35}$

\section{Anthropometrics}

Trained staff, following standardized procedures, measured parent and child heights (to the nearest $0.1 \mathrm{~cm}$ ) and weights (to the nearest $0.1 \mathrm{~kg}$ ). ${ }^{36}$ Two height and weight measures were averaged for each parent and child. Age- and gender-specific BMI $z$-scores for each child were calculated. Children were classified into underweight (less than fifth percentile), healthy weight (fifth to $<85$ th percentile), overweight $(\geq 85$ th to $<95$ th percentile), and obese ( $\geq 95$ th percentile) according to Centers for Disease Control and Prevention standards. ${ }^{17}$ Body mass index scores were calculated for the parent and classified as low and normal weight ( $<24.9)$, overweight $(\geq 25$ to $<30)$, or obese $(\geq 30) .^{18}$

Data Analyses. All analyses were intention-to-treat analyses-that is, data from mothers and children were analyzed on the basis of the condition to which they were initially assigned, regardless of the number of classes they attended (if any). This approach, although it reduces power, maintains the internal validity of the randomized controlled trial design. ${ }^{37}$ Because participants were nested within classes, intraclass correlations (ICCs) were first examined for each dependent variable to determine if a statistical approach controlling for such nesting was necessary. Because the ICCs were not zero, pretest to posttest changes for all outcome variables (except the categorical variables of feeding style and child weight status) were examined using multilevel analyses with the SPSS version 27 Mixed Models Program (IBM Corporation, 2020). Unfortunately, the SPSS Mixed Model Program does not provide measures of effect sizes; however, 95\% confidence intervals (CIs) were included for precision. Children's sex, children's age in months, and child BMI $z$-scores at pretest were control variables in all of the multilevel analyses. Significant condition $\times$ time interactions were followed up with simple main effects analyses and multilevel analyses comparing pairs of time points. The main analyses were conducted on participants who had data at all 4time points $(n=107-120)$. Paired comparisons used all available data ( $n=143-209)$. For the parent measures, the average percent of missing data across time points was $1.3 \%$ for the CEBQ and less than 1\% for the remaining questionnaire measures. Most of the missing data was addressed by calculating the subscale scores using the mean of the nonmissing items in that particular subscale. If greater than $25 \%$ of a subscale's items were blank, then the score was considered missing. The missing data percentages were higher for the child variables: $6.6 \%$ for the compensation trials, $4.0 \%$ for $\mathrm{EAH}$, $3.1 \%$ for the tasting task, and $2.4 \%$ for child BMI. The multilevel models also were rerun examining (1 at a time) possible program moderators: child age, child sex, child BMI status, and location (Houston vs Pasco). Moderation was tested by examining the significance of the moderator $x$ condition $\times$ time interactions.

Finally, to examine dosage effects, the multilevel analyses were rerun for those variables showing significant condition $\times$ time interactions.
In these analyses, only data from mothers in the prevention conduction at posttest, 6-months, and 12months were examined because the authors wanted to determine if the number of sessions mothers attended predicted their subsequent responses on the questionnaire assessments (the pretest assessments were not included in these analyses because these sessions occurred before mothers attended any classes). In conducting these analyses, the condition variable was replaced by a variable assessing the number of lessons attended (values ranged from 0 to 7 ). To determine if the dosage effects varied by time point, a separate set of analyses included the lessons attended $\times$ time interaction as a predictor (along with the corresponding main effects).

The effects of the program on mothers' feeding style were examined with 2 condition $\times$ feeding style Pearson $\chi^{2}$ tests -1 at pretest and 1 at posttest. Finally, a multinomial logistic regression was used to examine the effects of the program on the child BMI category (ie, healthy weight, overweight, and obese). The 4 children with underweight at pretest were excluded from this analysis. This regression examined the main effect of condition, controlling for the same variables as in the multilevel analyses (ie, child sex, child age in months, and child BMI $z$-score at pretest).

To control for type I errors, the critical $P$ values for each assessment were determined with the unweighted Bonferroni method, ${ }^{38}$ dividing the critical value of $P<0.05$ by the number of comparisons conducted for a given assessment. As discussed in Shaffer, ${ }^{35}$ corrections for familywise error involve correcting alphas separately for each family of hypotheses. Although defining families is a rather straightforward process in a simple experiment (eg, 1 family in a study with a single dependent variable and 1 independent variable with several levels), Shaffer argues that defining families becomes more complicated (and potentially more problematic) in large, more complex studies in which alpha corrections can substantially lower statistical power (ie, increase type II error). To 
balance concerns about type I and type II error in the current study, a family was therefore defined as the set of measures derived from a particular outcome assessment, and an alpha correction procedure was then used (the unweighted Bonferroni) to control for error rates within families. The resulting critical $P$ values were: FPI, $0.05 / 16=0.003 ;$ CFSQ , 0.05/ $3=0.017$; FKQ $0.05 / 6=0.008$; compensation trials, $0.05 / 1=0.05$; $\mathrm{EAH}$, $0.05 / 1=0.05 ; \quad \mathrm{FPQ}, \quad 0.05 / 2=0.025$; CEBQ, 0.05/8 = 0.006; and BMI, 0.05/ $2=0.025$. Only results that met these critical values are reported in the primary analyses. To minimize the probability of type II error, these critical values were applied only to $P$ values for the simple main effects analyses of time given the limited power of condition $\times$ time interactions in this type of design. ${ }^{39}$

Finally, exploratory analyses were run on variables that showed condition $\times$ time interactions in the prepost analyses reported by Hughes e $\mathrm{al}^{16}$ but did not show significant interactions here. Because the smaller samples at follow-up may have reduced the power to find significant time $\times$ condition interactions at 6 and 12 months, simple main effects analyses of time were run separately for the control and prevention groups to determine if the condition effects found earlier prepost analyses $^{16}$ persisted for these variables.

\section{RESULTS}

Three overall outcome areas were targeted as part of the efficacy of the SEEDS prevention program: parental feeding (practices, styles, and knowledge), child eating behaviors (self-regulation, trying new foods, and fruit and vegetable consumption), and child BMI. Significant outcomes were seen in various forms of parental feeding (changes in feeding practices, greater knowledge of feeding practices, higher feeding efficacy, and less uninvolved feeding styles). No changes were seen in child eating behaviors; however, children in the prevention group were less likely to be classified as overweight than the control group at the 12month follow-up. Details of these results are described below.

\section{Characteristics of the Sample}

Characteristics of the sample are presented in Table 1. Mothers were primarily first-generation immigrants $(>90 \%)$ with a wide range of educational backgrounds. About two thirds of the mothers were married, and about one third were employed outside of the home. Most mothers were overweight or obese (84\%). Thirtyeight percent of the children were overweight or obese. Statistical comparisons of mothers who participated at all 4 time points with those who did not participate at 1 or more time points ( $t$ tests and $\chi^{2}$ tests) on the pretest variables (ie, demographics and dependent variables) showed only 1 significant difference $(P<0.05)$ out of 48 comparisons, fewer than expected by chance alone.

Statistical comparisons ( $t$ tests and $\chi^{2}$ tests) of data from the 2 locations showed that mothers in the urban city were more educated $\left(\chi^{2}[7]=34.71, P<0.001\right)$, that their children were younger $(t[252]=-4.14$, $P<0.001)$, and that mothers in the agricultural community were more likely to be born in Mexico $\left(\chi^{2}[2]=28.67, P<0.001\right)$. Finally, $t$ and $\chi^{2}$ tests showed that the control and prevention groups did not significantly differ $(P<0.05)$ on any of the demographic variables or dependent variables at time 1 .

\section{Preliminary Analyses}

Examination of the distributions of the continuous dependent variables at pretest (with the exception of the tasting variables-see below) showed that parent roles showed limited variability (ie, $84 \%$ to $91 \%$ of mothers identified either 5 or 6 of the parent roles) and 2 variables required dropping a single outlier each (ie, number of fruits tried and COMPX). Because the number of kilocalories eaten in the absence of hunger was positively skewed, a natural logarithm transformation was performed for the analyses. Examination of the ICCs for the dependent variables at the pretest showed a wide range of values (from 0.00 to 0.108 ). The mean intraclass correlation for the pretest variables was rather low (mean $[\mathrm{M}]=0.014$ ); only 4 of 32 ICCs were $>0.030$ : kcal consumed in the absence of hunger $(0.108)$, the number of fruits tried on the FPQ (0.048), knowledge of the correct number of servings on the FKQ (0.036), and restriction on the FPI (0.032). Despite these low ICCs, multilevel analyses were used whenever possible (ie, for all variables except the categorical analyses of feeding style and weight status) to account for the nesting of participants within classes to yield accurate estimates of the significance values. The relatively high ICC for EAH may have reflected the operation of social factors in disinhibiting the eating of snacks during the EAH task. Although children could not see each other during this task (they were assessed in separate areas in the classroom through the use of portable screens), they could often hear one another so that social factors may have been operating. It is also possible that minor differences between the sessions in how the task was administered (eg, how many children were assessed at one time, minor deviations in the administration of the protocols) could have led to correlated outcomes within classrooms.

Using statistics from the current sample, power analyses using $\mathrm{G}^{\star}$ Power software (version 3.1.9.4; Department of Psychology, University of Düsseldorf, 2019) identified the observed power of detecting a significant condition $\times$ time interaction in a repeated analysis of variance with an alpha level of $P<0.05,2$ conditions and 4 measurements over time. Analyses were run separately when the mean correlations between the time points ranged from 0.06 (COMPX) to 0.94 (BMI Z-score). Besides these 2 outliers (and restriction, $r=0.30$ ), the mean correlations across the 4-time points for the outcome variables ranged from 0.41 to $0.69(\mathrm{M}=0.54)$. The sample size used in the power analyses was 107, the smallest sample size for a 4-time point analysis. This sample size was adjusted to account for an average ICC of 0.014 within classes in this nested design. These analyses showed that for all of the continuous outcome variables, the power to detect a small effect size $(f=0.15)$ was $81 \%$ or higher for the 4-time point 
analyses (except for the COMPX analysis in which power was $68 \%$ ).

Finally, because mothers were nested within classes, preliminary multilevel analyses were run to see if program effects varied by class. This involved examining the class by condition by time interaction for the various outcome variables. This interaction was not significant for any of these analyses.

\section{Number of Lessons Attended}

The mean number of lessons attended by mothers in the prevention group was 5.17 out of $7(\mathrm{SD}=2.11)$ : $35 \%$ of mothers attended all 7 lessons, $24 \%$ attended 6 lessons, $14 \%$ attended 5 lessons, $7 \%$ attended 4 lessons, and the number of mothers attending $0,1,2$, or 3 lessons was about $5 \%$ each. The number of lessons attended did not significantly differ as a function of location ( $F$ $[1,252]=1.00$, not significant).

Parent Feeding (Practices, Styles, and Knowledge)

Food Parenting Inventory. The multilevel analyses on the 16 Food Parenting Inventory (FPI) subscales showed significant condition $\times$ time interactions $(P<0.05)$ for 5 subscales (Table 2): encourage exploration of new foods, repeated presentation of new foods, serves measured portions, child involvement in food preparation, and responsiveness to child's fullness cues. For all 5 variables, the simple main effects analyses for the prevention group met the critical value of $P<0.003$, the control mothers showed no significant changes over time, and the prevention mothers showed significant increases from pretest to posttest with means at 6 and 12 months significantly higher than the pretest means. For 4 variables, the increases at posttest were maintained over time, with no significant changes from posttest to 12 months. Responsiveness to children's fullness cues significantly decreased from posttest to 12 months, although the mean at 12-months was still significantly higher than the pretest mean.
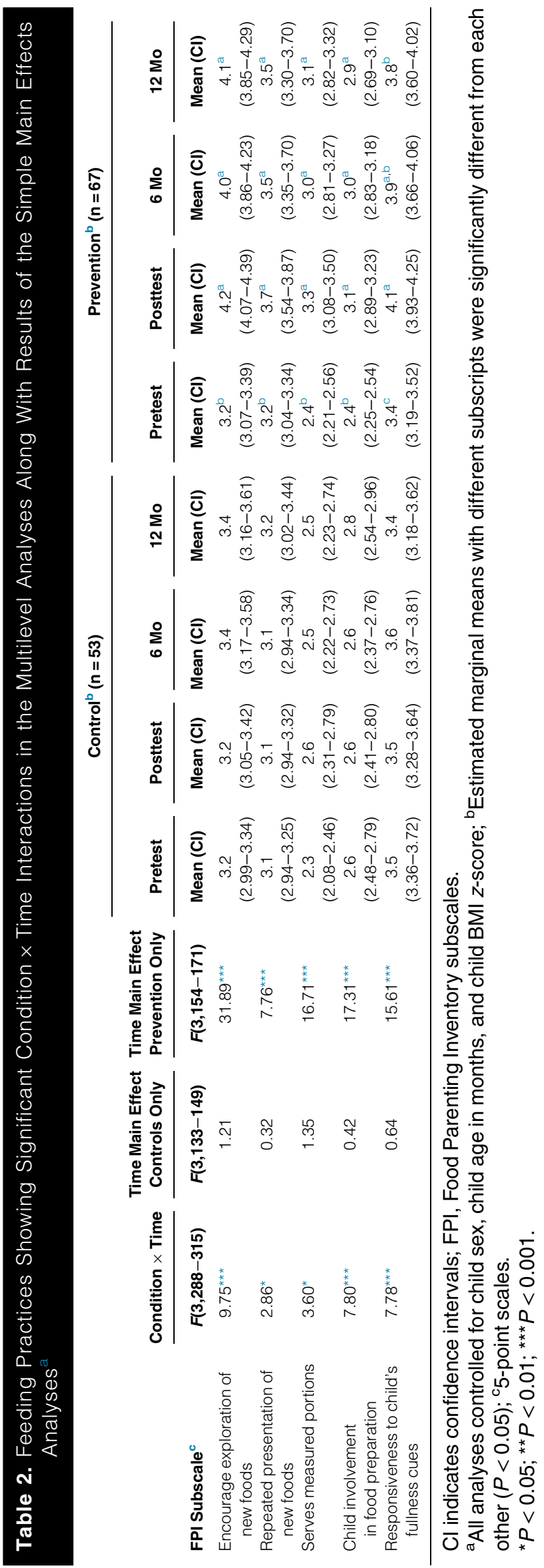
Caregiver's Feeding Style Questionnaire.

The multilevel analyses showed no significant condition by time interaction for demandingness $(F$ $[3,300.58]=0.67, P=0.57)$, but a significant interaction for responsiveness $(F[3,307.85]=5.65, P<0.001)$. Significant main effects analyses showed that responsiveness did not significantly change from pretest to posttest for control mothers (F $[3,134.38]=1.40, P=0.24)$, but significantly increased from pretest to posttest for prevention mothers ( $F$ $[3,167.47]=16.82, P<0.001)$ and this increase was maintained over time. This is shown in the estimated marginal means and 95\% CIs: pretest ( $\mathrm{M}=1.14$ [95\% CI, 1.11-1.17]), posttest $(\mathrm{M}=1.30$ [95\% CI, 1.27-1.33]), 6 months $\quad(\mathrm{M}=1.25 \quad[95 \% \quad \mathrm{CI}, \quad 1.21$ $-1.29])$, and 12 months $(\mathrm{M}=1.24$ [95\% CI, 1.20-1.28]). The posttest, 6 month, and 12 month means were significantly higher $(P<0.05)$ than the pretest mean, but did not significantly differ from each other. The proportion of mothers showing the 4 feeding styles did not differ at pretest $\left(\chi^{2}[3]=0.86, P=0.84\right)$, although they did differ at posttest $\left(\chi^{2}[3]=12.30, P\right.$ $<0.01)$. Follow-up $\chi^{2}$ tests showed that the only significant difference at posttest was for uninvolved mothers $\left(\chi^{2}[1]=6.84, P<0.01\right)$ with more control mothers showing the uninvolved feeding style (20.2\%) than prevention mothers $(7.8 \%)$. The proportion of the mothers showing the 4 feeding styles did not significantly differ at 6 months $\left(\chi^{2}[3]=1.92, P=0.59\right)$ or at 12 -months $\left(\chi^{2}[3]=4.52, P=0.21\right)$.

Feeding Knowledge Questionnaire. The multilevel analyses on the 6 Feeding Knowledge Questionnaire (FKQ) measures showed 5 significant condition $\times$ time interactions $(P<0.05)$ in which the $P$ value for either of the simple main effects met the critical value of $P<0.008$ (Table 3). Only efficacy away from home did not show significant effects. The simple main effects analyses showed that mothers in the control group showed no significant change over time, whereas mothers in the prevention group significantly increased or decreased from pretest to posttest, and these changes (except for a single variable) were maintained over time. The only
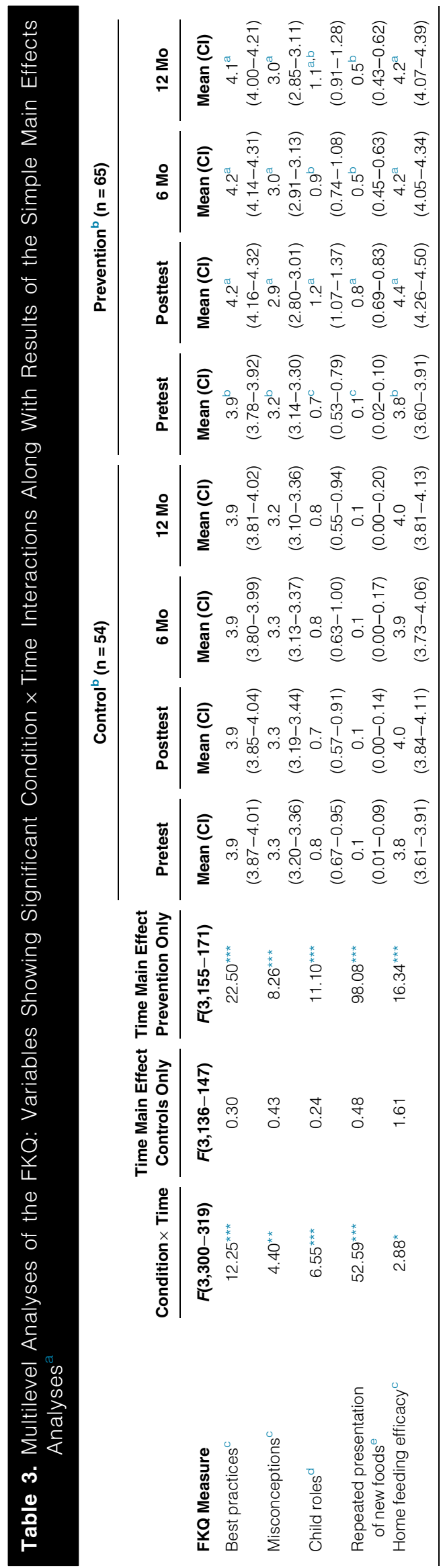

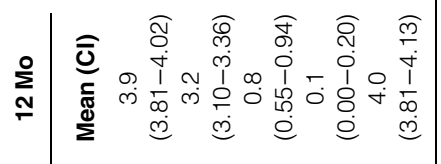

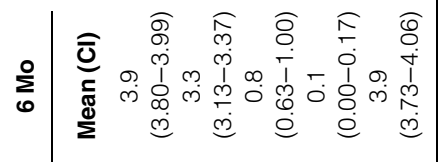

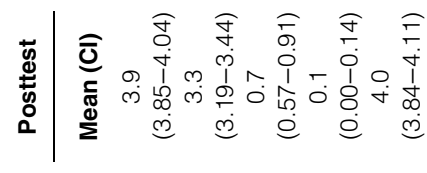

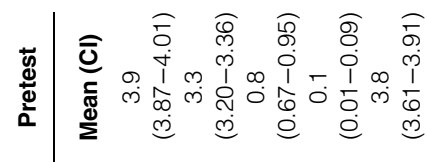

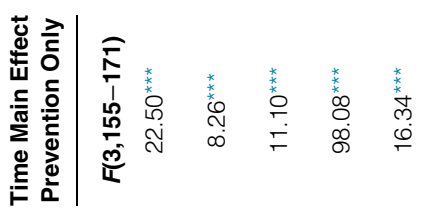

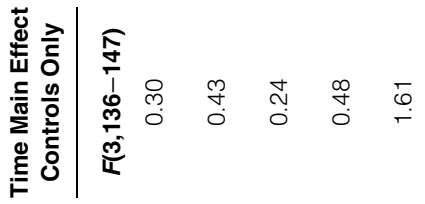

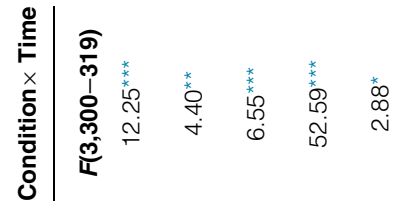

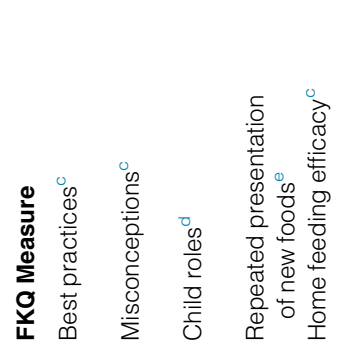

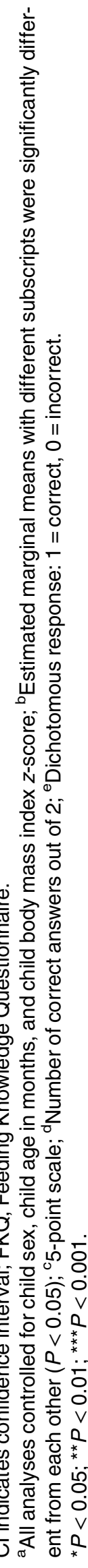


exception was knowledge about the number of repeated presentations of new foods. This score increased from pretest to posttest but then declined to an intermediate level at 6 and 12 months. However, the means at these last 2-time points were still significantly higher than the pretest mean. Inspections of the means in Table 3 show that prevention mothers significantly increased on knowledge of best practices, child roles, repeated presentation of new foods, and home feeding efficacy, and significantly decreased on feeding misconceptions.

\section{Children's Eating Behavior Outcomes (Self-regulation, Trying New Foods, and Fruit and Vegetable Consumption)}

Compensation protocol. The multilevel analysis on the COMPX scores measuring child eating self-regulation showed no significant condition $\times$ time interaction $(F[3,299.78]=0.44, P=0.72)$. On the basis of the Hughes et $\mathrm{al}^{16}$ prepost results, a concern was that this variable did not show much consistency over time; therefore, the correlations across time points for this variable were examined for the control group. The 6 correlations ranged from 0.01 to 0.26 , with a mean of 0.09 . Only 1 correlation was statistically significant, posttest to 12 months $(r[57]=0.26$, $P=0.05)$. The correlations for the prevention group ranged from -0.12 to 0.19 , none reaching statistical significance.

Eating in the absence of hunger. The condition by time interaction was not significant for kcal eaten in the absence of hunger $(F[3,337.88]=0.72$, $P=0.54)$. Unlike the compensation trials, children showed considerable stability in their responses to this task. The 6 correlations for the control group between time points ranged from 0.14 to 0.70 with a mean of 0.40 ; correlations for the prevention group ranged from 0.47 to 0.73 with a mean of 0.58 .

Children's Eating Behavior Questionnaire. The condition $\times$ time interaction was not significant for any of the CEBQ subscales measuring child eating self-regulatory behaviors.

Child tasting panel observation. Unfortunately, the tasting panel data did not provide sufficient variability in child responses to assess the impact of the SEEDS program. Individual differences in willingness to try new foods could not be assessed because the vast majority of foods were tried by virtually all of the children at pretest. The tasting rates for the 9 foods at pretest ranged from $90.3 \%$ to $97.2 \%$, with a mean of 94.6\%. Therefore, the tasting panel data were not analyzed further.

Food Preferences Questionnaire. The condition $\times$ time interaction was not significant for either the total number of fruits tried $(F[3,307.90]=1.02$, $P=0.38)$ or the total number of vegetables tried $(F[3,302.15]=1.00, P=0.39)$.

\section{Anthropometrics}

Child BMI z-score and weight status. The multilevel analysis on child BMI z-score showed no significant condition $\times$ time interaction $(F$ $[3,309.37]=0.18, \quad P=0.91)$. Child BMI $z$-scores were highly stable over time. The correlations between time points were: pretest to 6-months ( $r$ $[160]=0.94, P<0.001)$, pretest to 12 months $(r[145]=0.92, P<0.001)$, and 6 to 12 months $(r[125]=0.95, P$ $<0.001)$.

However, the multinomial logistic regressions on the children's 3 weight status categories showed significant effects of condition at 12 months. Although both the 6-month $\left(\chi^{2}[8]=204.09, P<0.001\right)$ and the 12-month $\left(\chi^{2}[8]=155.03, P<0.001\right)$ regressions were significant (primarily because of the pretest BMI z-score covariate), the only significant condition effect was at 12 months $\left(\chi^{2}[2]=7.76, P<0.005\right)$. In the 12 month analysis, examination of the odds ratios showed that when controlling for the covariates, children in the control group were more likely to be classified as overweight $(B=4.12, \quad P<0.05)$ or obese $(B=11.84, P<0.05)$ than children in the prevention group. As stated earlier, the percentage of children in the
3 weight status categories at pretest did not vary by condition $\left(\chi^{2}[2]=2.44, P=0.30\right)$.

\section{Program Moderators and Exploratory Analyses}

The moderating analyses for child age, child sex, child obesity status, and location showed no significant condition by time by moderator interactions. For the exploratory simple main effects analyses of the 4 variables showing significant condition $\times$ time interactions in the Hughes et $\mathrm{al}^{16}$ analyses but not here, the simple main effects of time were not significant for any variable for the control participants $(F<1.0)$, but were significant for the prevention participants: offer new foods ( $F$ $[3,154.71]=6.60, P<0.001)$, pressure to eat $(F[3,158.21]=3.56, P<0.05)$, food as a reward $(F[3,161.68]=4.12$, $P<0.01)$, and the number of vegetables tried $(F[3,154.61]=6.55, P<$ $0.001)$. Follow-up tests showed that for all except pressure to eat, these variables showed a significant change from pretest to posttest that was maintained at 6 and 12 months: offer new foods and number of vegetables tried increased and using food as reward decreased. Pressure to eat showed a significant decrease from pretest to posttest but increased at 6 and 12 months to values that did not differ significantly from each other or the pretest scores.

\section{Dosage Analyses}

The multilevel analyses examining the dosage effects showed that for 5 dependent variables, the number of lessons mothers in the prevention condition attended was positively and significantly associated with the maternal outcomes: encourage exploration of new foods ( $F$ $[1,84.03]=24.54, P<0.001)$, serves measured portions $(F[1,87.09]=9.92$, $P<0.01)$, knowledge of best practices $(F[1,84.80]=12.34, \quad P<0.001)$, knowledge regarding the repeated presentation of new foods $(F[1,85.98]$ $=7.45, P<0.01)$, and home feeding efficacy $\quad(F[1,85.77]=15.59, \quad P<$ $0.001)$. None of the dosage $\times$ time interactions were significant. The variables showing no significant dosage 
effects were: (1) FPI-repeated presentation of new foods, child involvement in food preparation, and responsiveness to child's fullness cues; (2) FKQ-misconceptions and child roles; and (3) CFSQ-responsiveness.

\section{DISCUSSION}

The most important effect found in this first efficacy trial of SEEDS was the reduced likelihood of Hispanic children having overweight or obesity at the 12-month follow-up for those who participated with their mothers in the prevention arm of the program. This effect was predicted for the prevention children compared with the control children. Few programs have targeted both Hispanic parents and their child within the same prevention program aimed at reducing obesity among this population. Specifically, in reviewing the literature, only 2 family-centered programs with 12-month assessments were found that attempted to reduce obesity in preschool-aged Hispanic children. ${ }^{12}$ Barkin et $\mathrm{al}^{13}$ found no differences in child BMI at any follow-up assessments on the basis of their program. However, Foster et $\mathrm{al}^{14}{ }^{14}$ in a small pilot intervention program with children whose BMI $z$-scores were greater than the 95th percentile, found no differences by randomization group (parent vs community health worker) but showed a reduction in BMI $z$-scores across all participants. A larger study is needed to determine the best approach (parent or community) to reduce childhood obesity. ${ }^{14}$ The SEEDS study informs the emerging literature on family-centered obesity prevention programs by showing that Hispanic preschoolers involved in the SEEDS prevention program were at a healthier weight at the 12-month follow-up than control children.

As expected, the SEEDS prevention program also showed changes in parental feeding behaviors at the 12month follow-up. Mothers increased (1) multiple presentations of new foods, (2) measured child portion sizes, (3) child involvement in food preparation, (4) responsiveness in their feeding approaches, (5) knowledge of best feeding practices, and (6) feeding efficacy. Mothers also showed reduced feeding misconceptions and less uninvolved feeding styles. These findings were also found at posttest ${ }^{16}$; parental feeding behaviors were maintained through the 12 month assessments. No increases in maternal structure during mealtime (ie, family meals) were seen at the 12month follow-up. Dosage predicted maternal outcomes for some variables as well. Maternal attendance ranged from 0 to 7 lessons $($ mean $=5.2)$, and mothers who attended more sessions were more likely to encourage the exploration of new foods, to serve measured portions, to demonstrate greater feeding knowledge, and to report higher levels of feeding efficacy in the home.

Only a few prevention programs have focused on parental feeding as a mechanism to promote healthier child weight status. For example, Frenn et $\mathrm{al}^{38}$ designed a prevention program aimed at promoting authoritative feeding and parental modeling to improve child diet and BMI percentile across a diverse ethnic group of middle school-aged children. Direct effects of authoritative feeding were found on weight showing lower child BMI percentile at the end of the study. In another obesity prevention program targeting parents of children aged 9-13 years, a curriculum was developed to teach better parenting practices, improve parenting styles, and increase parental self-efficacy. ${ }^{39}$ Although this curriculum showed promise, results of the curriculum on child BMI have not been published as of yet. Programs targeting older children may be limited because food preferences develop at earlier ages, ${ }^{4}$ and it may be more productive to focus on younger children during the time that preferences for healthier and less healthy foods are developing (eg, preschoolers). One recent study showed that changes in parental feeding practices predicted changes in child food intake (mean age 5 years). The changes in feeding practices did not result in a change in child weight status. ${ }^{40}$

It is interesting that the program produced no documented effects on child eating behaviors as predicted. Because the compensation and $\mathrm{EAH}$ tasks (observed eating self-regulation) and the child tasting panel (observed willingness to try new foods) have not commonly been used with Hispanic children from families with low incomes, these measures may not be sufficiently sensitive to detect individual differences in child eating behaviors in this population. As discussed in the paper detailing the short-term effects of the SEEDS prevention program, ${ }^{16}$ these measures may not have shown significant effects because of child reactions to adult demands in a group setting and cultural differences in responding. Furthermore, no program effects were found on maternal reports of child eating behaviors in this study despite using a commonly known and widely accepted assessment tool (CEBQ). ${ }^{28}$ A strong genetic component has been documented across child appetitive traits as measured by the CEBQ ${ }^{41,42}$ which may diminish the effects of the intervention.

The program did not have an impact on 2 areas of feeding (restriction and monitoring) and a commonly targeted aspect of family structure (family meals). Review articles have shown that prevention programs targeting less restriction, more monitoring, and higher frequency of family meals may protect against childhood obesity. ${ }^{43,44}$ Unfortunately, because of limitations on the amount of material that could be covered in 7 lessons, restriction and monitoring were not addressed in the curriculum, and only part of a single lesson was devoted to family meals.

In summary, family-based prevention programs have shown limited evidence of success in reducing childhood obesity, especially those targeting parental feeding behaviors among Hispanic parents of preschoolers. One unique aspect of the SEEDS program was the use of highquality videos as part of a teaching mechanism in each of the 7 lessons. These videos were developed with the sole purpose of fostering internalization of the values and behavioral patterns introduced in the program. The introduced constructs were further endorsed by using members of the community along with actors of 
similar ethnicity in the videos. Furthermore, after watching the videos, mothers engaged in group-based, experiential learning activities to reinforce the concepts introduced in the videos. Given the important role of parents in fostering a healthier child weight status, especially in families with limited resources, the SEEDS program has the potential to reduce childhood obesity by targeting parental feeding behaviors that are amenable to change.

The findings should be considered in light of the study's limitations. The program was implemented in only 2 locations, the classes were offered only in Spanish, the vast majority of participants were firstgeneration immigrants, the maternal assessments were self-report, the fidelity observations were scheduled on the basis of observer availability (rather than randomly chosen), and the results of this study raise questions about the utility of some child eating measures for Hispanic children from families with low incomes. Additional limitations include that participants were paid to participate in the study (it is likely that lower participation rates would have occurred without financial incentives), and the control arm received no curriculum, so there was no control for attention effects. ${ }^{45,46,47}$ Finally, although the pretest sample had sufficient power to demonstrate program effects, subject attrition at the 6- and 12-month follow-ups lowered the power to find some program effects and to identify the impact of program moderators. However, the finding that the mothers who did not participate at all 4-time points did not differ in any systematic way from those who completed all 4-time points reduces concerns about the impact of attrition rate on the validity of the results.

\section{IMPLICATIONS FOR RESEARCH AND PRACTICE}

Among previous family-based prevention programs targeting Hispanic families, few programs have focused on preschoolers with even fewer showing effects on child weight status. ${ }^{14}$ This study provides evidence for the 12-month efficacy of the SEEDS childhood obesity prevention program in providing changes in maternal feeding behaviors as well as healthier weight status for children. Changes in child weight status were potentially a consequence of changes in maternal feeding practices and knowledge resulting from the program. This information may be helpful to researchers who are developing tailored programs for families with low resources, especially among Hispanic families. Replication of these results in future studies would support the efficacy of the program; implementing the program in other populations would support efficacy as well. No effects were found on child eating behaviors; however, future studies that include better measurement of child eating behaviors and account for cultural differences could produce different results. Given the labor-intensive nature of the child lessons, examining whether the inclusion of the child lessons has an impact on child outcomes over and above the parent lessons alone could be beneficial. Given the importance of parents in the development of child eating behaviors, the results of the current study show that a family-based approach can lead to positive outcomes in addressing the problem of childhood obesity.

\section{ACKNOWLEDGMENTS}

This research is supported by funds from the US Department of Agriculture (Grant USDA 2011-68001-30009). This work is also a publication of the US Department of Agriculture (USDA/ ARS) Children's Nutrition Research Center, Department of Pediatrics, Baylor College of Medicine (Houston, TX) and funded, in part, by the USDA/ARS (Cooperative Agreement 58-3092-0001). The authors would like to thank the Head Start families who participated in this study, as well as the personnel at Head Start, whose support has been invaluable.

\section{REFERENCES}

1. Fryar CD, Carroll MD, Ogden CL. Division of Health and Nutrition Examination Surveys. Prevalence of Overweight, Obesity, and Severe Obesity Among
Children and Adolescents Aged 2-19 Years: United States, 1963-1965 Through 2015-2016. National Center for Health Statistics; 2018.

2. Hales CM, Fryar CD, Carroll MD, Freedman DS, Ogden CL. Trends in obesity and severe obesity prevalence in US youth and adults by sex and age, 2007-2008 to 2015-2016. JAMA. 2018;319:1723-1725.

3. Ogden CL, Carroll MD, Kit BK, Flegal KM. Prevalence of childhood and adult obesity in the United States, 2011 -2012. JAMA. 2014;311:806-814.

4. Birch LL, Ventura AK. Preventing childhood obesity: what works? Int J Obes (Lond). 2009;33(suppl 1):S74-S81.

5. Amini M, Djazayery A, Majdzadeh R, Taghdisi MH, Jazayeri S. Effect of school-based interventions to control childhood obesity: a review of reviews. Int J Prev Med. 2015;6:68.

6. Showell NN, Fawole O, Segal J, et al. A systematic review of home-based childhood obesity prevention studies. Pediatrics. 2013;132:e193-e200.

7. Wang Y, Wu Y, Wilson RF, et al. Childhood Obesity Prevention Programs: Comparative Effectiveness Review and MetaAnalysis. Report No: 13-EHC081-EF. Agency for Healthcare Research and Quality (US); 2013.

8. Mikkelsen MV, Husby S, Skov LR, Perez-Cueto FJ. A systematic review of types of healthy eating interventions in preschools. Nutr J. 2014;13:56.

9. Ash T, Agaronov A, Young T, Aftosmes-Tobio A, Davison KK. Familybased childhood obesity prevention interventions: a systematic review and quantitative content analysis. Int J Behav Nutr Phys Act. 2017;14:113.

10. Gentile N, Kaufman TK, Maxson J, et al. The effectiveness of a family-centered childhood obesity intervention at the YMCA: a pilot study. J Commun Med Health Educ. 2018;8:591.

11. Gray LA, Hernandez Alava M, Kelly MP, Campbell MJ. Family lifestyle dynamics and childhood obesity: evidence from the millennium cohort study. BMC Public Health. 2018;18:500.

12. Tamayo MC, Dobbs PD, Pincu Y. Family-centered interventions for treatment and prevention of childhood obesity in Hispanic families: a systematic review. $J$ Commun Health. 2021;46:635-643

13. Barkin SL, Heerman WJ, Sommer EC, et al. Effect of a behavioral intervention for underserved preschool-age children on change in body mass index: a 
randomized clinical trial. JAMA. 2018;320:450-460.

14. Foster BA, Aquino CA, Gil M, Gelfond JA, Hale DE. A pilot study of parent mentors for early childhood obesity. J Obes. 2016;2016:2609504.

15. Hughes SO, Power TG, Beck A, et al. Strategies for Effective Eating Development-SEEDS: design of an obesity prevention program to promote healthy food preferences and eating self-regulation in children from low-income families. $J$ Nutr Educ Behav. 2016;48:405-418.

16. Hughes SO, Power TG, Beck A, et al. Short-term effects of an obesity prevention program among low-income Hispanic families with preschoolers. $J$ Nutr Educ Behav. 2020;52:224-239.

17. Centers for Disease Control and Prevention. Defining childhood obesity. https://www.cdc.gov/obesity/childhood/defining.html Accessed August 27, 2020.

18. Centers for Disease Control and Prevention. Defining adult overweight \& obesity. Updated April 28, 2021. https://www.cdc.gov/obesity/adult/ defining.html. Accessed May 14, 2021.

19. Goodell LS, Johnson SL, Antono AC, Power TG, Hughes SO. Strategies lowincome parents use to overcome their children's food refusal. Matern Child Health J. 2017;21:68-76.

20. Harris County Department of Education Head Start. What does the HCDE Head Start program offer your children? https://www.hcde-texas.org/ Page/9. Accessed May 14, 2021.

21. Power TG, Johnson SL, Beck AD, Martinez AD, Hughes SO. The Food Parenting Inventory: factor structure, reliability, and validity in a low-income, Latina sample. Appetite. 2019;134:111-119.

22. Hughes SO, Power TG, Fisher JO, Mueller S, Nicklas TA. Revisiting a neglected construct: parenting styles in a child-feeding context. Appetite. 2005;44:83-92.

23. Hughes SO, Power TG, Papaioannou MA, et al. Emotional climate, feeding practices, and feeding styles: an observational analysis of the dinner meal in Head Start families. Int J Behav Nutr Phys Act. 2011;8:60.
24. Hughes SO, Shewchuk RM, Baskin ML, Nicklas TA, Qu H. Indulgent feeding style and children's weight status in preschool. J Dev Behav Pediatr. 2008;29:403-410.

25. Johnson SL, Birch LL. Parents' and children's adiposity and eating style. Pediatrics. 1994;94:653-661.

26. Johnson SL, Taylor-Holloway LA. Non-Hispanic white and Hispanic elementary school children's self-regulation of energy intake. Am J Clin Nutr. 2006;83:1276-1282.

27. Johnson SL. Improving preschoolers' self-regulation of energy intake. Pediatrics. 2000;106:1429-1435.

28. Birch LL, Deysher M. Conditioned and unconditioned caloric compensation: evidence for self-regulation of food intake in young children. Learn Motiv. 1985;16:341-355.

29. Fisher JO, Birch LL. Restricting access to foods and children's eating. Appetite. 1999;32:405-419.

30. Lansigan RK, Emond JA, Gilbert-Diamond D. Understanding eating in the absence of hunger among young children: a systematic review of existing studies. Appetite. 2015;85:36-47.

31. Wardle J, Guthrie CA, Sanderson S, Rapoport L. Development of the children's eating behaviour questionnaire. $J$ Child Psychol Psychiatry. 2001;42:963-970.

32. Frankel LA, O'Connor TM, Chen TA, Nicklas T, Power TG, Hughes SO. Parents' perceptions of preschool children's ability to regulate eating. Feeding style differences. Appetite. 2014;76:166-174.

33. Sullivan SA, Birch LL. Pass the sugar, pass the salt: experience dictates preference. Dev Psychol. 1990;26:546.

34. Moding KJ, Bellows LL, Grimm KJ, Johnson SL. A longitudinal examination of the role of sensory exploratory behaviors in young children's acceptance of new foods. Physiol Behav. 2020;218:112821.

35. Skinner JD, Carruth BR, Bounds W, Ziegler PJ. Children's food preferences: a longitudinal analysis. J Am Diet Assoc. 2002;102:1638-1647.

36. Lohman TG, Roche AF, Martorell R. Anthropometric Standardization Reference Manual. Human Kinetics Books; 1998.
37. McCoy CE. Understanding the intention-to-treat principle in randomized controlled trials. West J Emerg Med. 2017;18:1075-1078.

38. Shaffer JP. Multiple hypothesis testing. Annu Rev Psychol. 1995;46:561-584.

39. Brookes ST, Whitely E, Egger M, Smith GD, Mulheran PA, Peters TJ. Subgroup analyses in randomized trials: risks of subgroup-specific analyses; power and sample size for the interaction test. J Clin Epidemiol. 2004;57:229-236.

40. Frenn M, Pruszynski JE, Felzer H, Zhang J. Authoritative feeding behaviors to reduce child BMI through online interventions. J Spec Pediatr Nurs. 2013;18:65-77.

41. Ruiter EL, Fransen GA, Molleman GR, van der Velden K, Engels RC. The effectiveness of a web-based Dutch parenting program to prevent overweight in children 9-13 years of age: study protocol for a two-armed cluster randomized controlled trial. BMC Public Health. 2015;15:148.

42. Somaraki M, Eli K, Sorjonen K, Ek A, Sandvik P, Nowicka P. Changes in parental feeding practices and preschoolers' food intake following a randomized controlled childhood obesity trial. Appetite. 2020;154:104746.

43. Wardle J, Carnell S. Appetite is a heritable phenotype associated with adiposity. Ann Behav Med. 2009;38(suppl 1):S25$\mathrm{S} 30$.

44. Wardle J, Carnell S, Haworth CM, Farooqi IS, O'Rahilly S, Plomin R. Obesity associated genetic variation in FTO is associated with diminished satiety. $J$ Clin Endocrinol Metab. 2008;93:36403643.

45. Dwyer L, Oh A, Patrick H, Hennessy E. Promoting family meals: a review of existing interventions and opportunities for future research. Adolesc Health Med Ther. 2015;6:115-131.

46. Ventura AK, Birch LL. Does parenting affect children's eating and weight status? Int $J$ Behav Nutr Phys Act. 2008;5:15.

47. Aycock DM, Hayat MJ, Helvig A, Dunbar SB, Clark PC. Essential considerations in developing attention control groups in behavioral research. Res Nurs Health. 2018;41:320-328. 